\title{
Population Characteristics and Institutional Participation: How Do Migrant Workers Make A Choice under Health Insurance Policy in China?
}

\author{
Yang Yifan, Mi Zengli \\ School of public administration, Southwestern Jiaotong University, Cheng Du, China \\ mizl606126@126.com, tuboyang@gmail.com
}

\begin{abstract}
On the base of Chengdu's survey data, this paper focuses on migrant workers' behavior of participating in the the urban insurance .This research use the Logistic regression model to analysis and do empirical study of the characteristic factors that affect the insured: health condition, income levels, job stability, and family issues. The result shows that the most important influencing factor for the migrant workers to participate in the urban medical insurance is the family issues followed by health conditions and job

Index Terms - migrant workers, medical insurance, influencing factors, system involvement
\end{abstract}

\section{Introduction}

With China's continues urbanization and industrialization, the migrate workers have gradually become one of the important part of the urban employment group. Most of them are engaged in high-risk and high labor intensity work and don't have medical insurance due to the difficulty of reimbursing medical expenses in the city, transferring medical insurance relationship and really returning to rural areas to participate in the new cooperative medical insurance. In recent years, although our government has been to expand the coverage of social insurance, including medical insurance and take it as the top priority of the social security work, hoping to eliminate the coverage "death corner" institutionally, some research findings reflect that even in the cities which is more developed and have established the medicare including migrant workers, the actual insurance rates and coverage is still far cry from the expected. The deep-seated reasons will directly relate the emphasis of extending the coverage of the national health insurance system for migrant workers: if the individual rational choice influence the migrant workers' participation most, then, it should focus on design the involvement incentive mechanism to attract migrant workers insured in policy design; if the demand for medical services is passively suppressed due to the objective factors, then, the policy design should focus on eliminating of weakening the restrictions, and release the reasonable medical needs. So far, the researches about migrate worker's medical insurance are mainly "grand narrative" in institutional level, lacking of the experience research for typical region and the behavior of typical group's involvement. Besides, it also lack empirical studies on the background of the urban-rural relations conversion and the overall development, and focus on the dynamic and reality relationship among individual, family and social system. Based on the obvious findings, the goal of this research is to study the survey data of balancing urban and rural development experimental zone - Chengdu migrant workers's participation mode and the effect factors of the enrollment of medical insurance system. In the study, we take the migrate workers not only as the institutional target group but the system participants to do the integration analysis. Though the consolidation of personal characteristics and unit characteristics, we explore the factors that influence the migrant workers' participation to the medical insurance system on the basis of the living condition, economic status, and family issues of migrate workers. This will further the understanding of the interactive logic mechanism between the migrate worker's medical security system and the behavioral strategy of enrollment both in theory and practical, and provide a reference for the government to improve the health insurance policy.

\section{The Research Hypothesis}

What are the factors that affect the migrant workers' choice in the enrollment of medical insurance? Different theoretical perspectives may come to a different focus. This article will take the medical insurance system as the practice of rules on the perspective of social theory. This means that the system object is not a completely passive taker. And the system is not a completely external constraints structure but has an inadvisable influence on people's behavior, which is the outcome of the diverse actors' action "structured" in the system's course of practice. In our opinion, if we can use the measurement methods to present the effect process and portray the main features of variable and policy background variables though the model, and at the same time focus on the analysis of the target groups' differentiation and differences, it is possible to study the difference of medical insurance system enrollment behavior and influence factors though the concrete practice of the different behavior subjects, and explain the different results of the medical insurance system applied to the migrate workers.

H1: there is a negative collection between the health condition of migrant workers and their medication insurance enrollment rate.

H2: there is a positive correlation relationship between the migrant workers' income and their medication insurance enrollment rate. 
H3: there is a positive correlation relationship between the migrant workers' work stability and their medication insurance enrollment behavior.

H4: there is a positive correlation relationship between the migrant workers' family issues and their medication insurance enrollment behavior.

\section{The Data Sources and Analysis Method}

\section{A. The data sources}

In order to obtain the research data, author conducted a questionnaire survey in Cheng Du, from August 15, 2011 to Feb 25 -2012, and a total of 3230 questionnaires were distributed. Those survey covered six districts and one county of Cheng Du, and has a certain representation: Cheng Du Railway Station (Cheng Hua District) a total of 480 people, Yu Hui talent labor market, Shudu yinghui human resource market (Jin Jiang District), a total of 500 people, Cheng Du high-tech zone human resource market (Wu Hou District) 400, cheng Du sands north bus station the station (Qing Yang District ) 520, Wen Jiang area passenger transportation center (Wen Jiang District) 470, Xin Du District human resource market (Xin Du District) 400, as well as the PI County human resources market (PI County) 460 people.

\section{B. Variables Measuring}

1) Dependent variables: This paper mainly discusses the influence factors of rural migrant workers to participate in medical insurance, the dependent variable is whether to participate in medical insurance. Obviously, this argument is a virtual variable, with the "join" to say.

$$
\text { join }=\left\{\begin{array}{l}
1 \\
0
\end{array}\right.
$$

Take "0" as a man has not participated in medical insurance, while take "1" as a man has participated in medical insurance.

2) Independent variable: Firstly, this argument includes such as gender, age, registered permanent residence and basic demographic index. Secondly, in order to validate hypothesis 1, we introduced a variable, "body circumstance (health_situation)". Its value is "0" said poor, while "1" said the general, and "2" said is very good. It is important to note that the variable of migrant workers by self evaluation and draw, not from professional medical institutions, so may cause this part of the data and the actual situation has certain deviation. In order to validate hypothesis 2 , we introduce the variable, "income (income)". Variables, "on-the-job time (working_period)" and "there is a labor contract or not (contract)", are used to describe hypothesis 3. Finally we introduce "family/ individuals (family)" this virtual variable to verify hypothesis 4 .

3) Measurement method: The method of measurement this article uses is the Logistic regression model, which is usually used to analyze two classification dependent variables. Logistic regression analysis can tell the influential factors from numerous suspicious factors affecting the events occurrence probability. The estimated model is as follows:

$$
\operatorname{Logistic}(p)=\ln \left(\frac{p}{1-p}\right)=\beta_{1}+\beta_{2 i} X_{i}+\varepsilon_{i}
$$

In the formula (1), $\beta_{1}$ is the intercept, $X_{i}$ is the independent variable mentioned above, $\varepsilon_{i}$ is the error term. Two kinds of data are usually used to estimate the data of Logistic regression model: a single case data, or data in the form of group. In this paper, as the data mainly comes from survey data, which means the capacity is limited, therefore, we will use a single case data (micro data) to carry on the analysis.

\section{The Empirical Results and Model Explanation}

Based on the descriptive statistical analysis of the variables, this paper conducted the Logistic regression analysis. The research result shows, the individual of migrant workers'self-evaluation on their health condition has a significant impact on whether to purchase medical insurance. Compared to the migrant workers of poor health self-evaluation results, the odd ratio of enrollment of the medical insurance of the "general",have decreased by 2.6260,and that of the "good" have decreased by 3.3322.This shows that the more positive the migrant workers'self-health evaluation are, the lower the probability of their enrollment in medical insurance. And the more positive the migrant workers' self-health evaluation are, the lower the probability of their enrollment in medical insurance. In fact, the result is consistent with the assumption of rational economic person in economics, which also shows that there is a certain degree of risk of adverse selection in the basic medical insurance. It also verified the assumption 1: there is a negative correlation relationship between the health condition of migrant workers and their medication insurance enrollment rate.

For the hypothesis test 2, in order to analyze this unusual outcome further, we take the wage income as subject of the sample and regroup to observe. The results indicate that the common characteristics of the high-income migrant workers epitomize in the industry, gender and family situation; the migrant workers at media income level maintain a high degree of consistency; and the he migrant workers at low income level have a high concentration in industry and gender. Further, we put other independent variables one by one into the fixed income items, and do the two-factor Logistic regression. we can see from table 1 that there is a significant correlation between the income and migrant workers enrollment of the medical insurance behavior. 
TABLE1 The Logistic test results of two factors

\begin{tabular}{|l|l|l|}
\hline & $\begin{array}{l}\text { Coefficient estimates } \\
\text { of income items }\end{array}$ & $\operatorname{Prob}(>|\mathbf{z}|)$ \\
\hline Income+gender & 0.0005509 & $0.027786 *$ \\
\hline Income+age & $5.732 \mathrm{e}-05$ & 0.67631 \\
\hline $\begin{array}{l}\text { Income+location of } \\
\text { registered resident }\end{array}$ & 0.0003243 & 0.139071 \\
\hline Income+family issues & 0.0000419 & 0.84579 \\
\hline Income+on-the-job time & -0.0012166 & $1.44 \mathrm{e}-14 * * *$ \\
\hline Income+industry & -0.0005066 & 0.0730. \\
\hline $\begin{array}{l}\text { Income+whether signed the } \\
\text { labor contract }\end{array}$ & -0.0007415 & $0.003462 * *$ \\
\hline Income+health condition & 0.0005509 & $0.027786 *$ \\
\hline
\end{tabular}

The table above shows that, in all significant test project, only gender and health situation change the coefficient estimates of the income from negative to positive. This shows that under the condition of taking the gender and health situation into account at the same time, the assumption 2 becomes valid. After careful analysis of the two items' data, we found that in the sample of 2790 people, there are 1030 females and 1760 males, and the female's 1,722.95 yuan. There are 20 female who enrolled the medical insurance, and their average income is 1726.65 yuan. The following table shows the relationship between female's health and enrollment of the medical insurance.

As a result, the regression results show that it's the negative negative correlation between the two factors which completely overturned the hypothesis 2.In order to analyze this unusual outcome further, we take the wage income as subject of the sample and regroup to observe. Further, we put other independent variables one by one into the fixed income items, and do the two-factor Logistic regression. The results are as follows, in all significant test project, only gender and health situation change the coefficient estimates of the income from negative to positive. This shows that under the condition of taking the gender and health situation into account at the same time, the assumption 2 becomes valid. After careful analysis of the two items' data, we found that in the sample of 2790 people, there are 1030 females and 1760 males, and the female's $1,722.95$ yuan. There are 20 female who enrolled the medical insurance, and their average income is 1726.65 yuan. The following table shows the relationship between female's health and enrollment of the medical insurance. In addition, the indicators of family/personal regression results show that compared to the "independence" migrant workers, the "family-type" migrant workers are more likely to purchase for the medical insurance ( $\beta=1.9700)$ ); and the probability is 7.17 times of the former. This shows that the assumption 4 is valid.

\section{Research Conclusions and Policy Recommendations}

Judging from the in-depth analysis of the results of migrant workers' participation in medical insurance, the three assumptions: self-attribution, job stability and the family issues are basically right. In accordance with the family background of the migrant workers in the sample, the author divided it into three categories. The first category is one of the family labor going out to work, in which the young male labor becomes one of the migrate workers and the other family members have other stable sources of income (For example, their parents have farming income and their wife operated a small grocery). The second category is the family labor force (both spouses) going out to work, and with the old and the children leaving at home in rural areas. The third category is the nuclear family members have moved into the city without the town registered resident. In short, on the issue of migrant workers' participation in the urban medical insurance system, it's neither an individual's freedom choice based on pure economic rationality, nor the unilateral discretion out of the individual, but a interaction result of the institutional environment, income constraints and family issues. In other words, this can be take as social computing based on the interest consideration of the whole family. And it shows that faced with the changing opportunities pattern in the period of urban and rural transformation and co-ordinate development, the migrate workers, as a special transitional group, presented a social behaviour with the feature of strategic, integrity and adaptability.

The above research results show that if we want to improve the coverage of migrant workers' participation in the urban medical insurance, we should consider the policy issues on following two aspects. First of all, pay attention to the impact of insurance system on migrant workers' participation in the urban medical insurance, which include the relational schema between the new rural cooperative medical insurance and the urban residents medical insurance, the medical urban workers insurance, and the issue of place changing and the alteration of the medical insurance during the migration between the rural and urban. Secondly, although there exist high homogeneity in the migrant worker group, yet, there's a significant difference in the participation of different medical insurance for the migrate workers with different backgrounds. Some institutional arrangements objectively weakened and even deprived part of the migrant workers' eligibility to participate in the insurance.

\section{References}

[1] Xie Yong, Li Fang. Empirical study on migrant workers' will to participate in the social insurance _Based on Nanjing. Population Research.3,63-70(2009)

[2] Liang Weiping, Zheng Jianzhong, He Lu, Zhang Xiaodan. Study on demand and utilization of health service for migrant workers in Tai-yuan. Chinese Health Resources.2,67-69 (2010)

[3] Song Jing, Leng Mingxiang, Meng Fan, Rong Chaodi. The Empirical Research in the Situation of the Migrant Workers'Health Service Utilization. Chinese Primary Health Care.5,10-11(2010)

[4] Guo Yu, Factors affecting migrant workers'participating in the urban social security system - empirical analysis on the use of Probit model. The World of Survey and Research.1,42-45(2011) 